\title{
Mandela effect \& Déjà vu: Are we living in a simulated reality?
}

This paper was downloaded from TechRxiv (https://www.techrxiv.org).

\section{LICENSE}

CC BY 4.0

SUBMISSION DATE / POSTED DATE

25-09-2021 / 29-09-2021

\section{CITATION}

Bhattacharjee, Deep (2021): Mandela effect \& Déjà vu: Are we living in a simulated reality?. TechRxiv. Preprint. https://doi.org/10.36227/techrxiv.16680904.v1

$\mathrm{DOI}$ 


\section{Mandela effect \& Déjà vu: Are we living in a simulated reality?}

If reality is being augmented as a simulation; then from the modern norm of physics, it is possible that everywhere around us, including us and what we perceive is a simulation simulated by a super powerful computer from a farfetched future times, taken the Einstein'an notion, that, past, present and future occur simultaneously. It is quite probable between two consecutive amplitudes that, the simulation can either be done by the supercivilization in $\geq$ Kardashev 3.0 scale or by some higher order entities existing in a dimensional domain beyond our perceiveness and notion of our understanding. To agree to the fact of the simulation hypothesis, there exists a mathematical foundation of the desired logic behind this simulation, which will be investigated throughout this paper whose another consequence might be the déjà vu or the Mandela effect. The errors arise in this simulation is a form of glitch in the matrix that should happen because of the commutable lagging of the super-intelligent computers of either future ones or higher-order ones. Preciseness about the calculations of dimensions opened a way for $t+s=2+10$ where the non-locality of the time being perceived as a 2-dimensional entity opens up the door for further investigations. The more will be discussed in detail in this paper.

INFINITY CONVERGES - Einstein's theory $^{\mathrm{a}}$ gave us a tantalizing hint about the versatility of the time that we perceive in reality. This concept has been put forward by the string theorists to split the $4^{\text {th }}$ dimensional time into $\mathrm{a} 4^{\text {th }}$ and $5^{\text {th }}$ dimensional time separated from space where time itself can be 2-dimensional ${ }^{b}$. This is not purely an artifact of any imagination, there are extensive mathematics behind this claim and also detailed logic as to why time can be perceived as a 2-dimensional entity? Einstein's theory teaches us that time being an 1-dimensional arrow can only move in forward direction as going backward in time would open up several sorts of dirty paradoxes that complexified even violated the real nature of the physical law. So, time in a sense that we perceive is moving forward with space, even if one calculates then the composite motion of the Earth, in the Milky Way galaxy is about 4.4 million kilometers per hour in this universe ${ }^{c}$. So, time couldn't be kept still, it is moving and moving in only forward direction. But, what we can deduce from it is that, theories that prohibits the travelling of oneself in backwards direction due to the presence of a chronal agent as depicted by Prof. S.W Hawking in his Chronology Protection Conjecture ${ }^{d}$. But, Prof. K.S. Throne later in his paper argues that its not obvious that, chronology is strongly protected all the time, rather there can be instances where chronology has been weakly protected allowing one to travel backwards in a wider limit $^{\mathrm{e}}$. But, physics remains fancy and its fictional world gives rise to several paradoxes $^{f}$ that are purely theoretical called Grandfather's paradox ${ }^{g}$, Bootstrap paradox', Polchinski's paradoxi. However, whatever the case might be, all these depict one point, the nonlocal properties of time. Now, what exactly do we mean by non-local time? The time that we individuals perceive is local where it appears only as an one dimensional arrow, where to every cause there is an associated events and causality holds strictly in principle. But, what if we try to perceive time not from one individual persons point of view, not even from multiple persons point of view, but from a larger arena, through the perspectives of this universe. Does we then get a hint that everything is in loops, say that time like all the 3 functions of time; Past, present and future are happening simultaneously in a closed loop that all are simultaneous except the fact that they are separated by a large gap of time which is impossible for humans to comprehend in this 4-dimensional reality. Thus allowing us to deduce that, time is a 2-dimensional closed loop rather than a 1-dimensional arrow that belongs to the reality and act in a simultaneous way as regards to the events happening in past, present and future.

Our universe being in a state of vacua where all the forces of the physical laws are perfectly balanced as to grander the birth of this stable universe is nothing rather than a single vacua floating among $10^{500}$ vacuas in the cosmic landscape as proposed by Prof. Susskind in the first place ${ }^{j}$. There is an inert tendency of nature that things tends to be alike when the infinity converges and there can be one possible universe almost same as ours with all the elements almost alike as ours including us doing the same things, as the iterations got ended and starts in a new place after the convergence of the infinity. So, does infinity really converges after a certain point rather than blowing out to further infinities and diverges. The Riemann Zeta function when plotted with the Ramanujan's hypothesis gives a surprising answer that is ${ }^{k}$;

$$
\zeta(s)=2^{s} \pi^{s-1} \sin \frac{\pi s}{2} \Gamma(1-s) \zeta(1-s)
$$

Where if $\operatorname{Re} s<1$, then the analytic continuation would be extend to an infinite series where the function evolves into;

$$
\zeta(-1)=\frac{1}{2 \pi^{2}}(-1)(1)\left(\frac{\pi}{\sqrt{6}}\right)^{2}=-\frac{1}{12}
$$

BREAD-SLICE TIME \& BLOCK THEORY - It is not difficult to imagine that the universe is a loaf of bread that is extending in one direction with the other direction remaining fixed. Then, the entire loaf would represent the past, present and future and each slice of the loaf represents a particular instance of space-timel. The slice can be taken orthogonally representing the events that are taking place in the arrow of time. Thus a slice at the beginning of the loaf

\footnotetext{
${ }^{1}$ Email: itsdeep@live.com
} 
represents a farfetched past while the slice at the direction of elongation represents present which when elongates represents the future. In this concept, if we are living in a particular slice then our ambient slice represents past and future which describes a continuity of events in a non-local timeline that represents our identity. Now, consider each slice as a cube. A 3-dimensional block with a 1dimensional time (locally) as a slice of time $^{m}$. This block will represent our world at the present instant of time. The block slowly moves forward through the loaf taking shape of a new block as time progresses, so as space thus concluding that, to move in time is to move in space. It is however crucial to note that the block will move only in one direction and reverse flow is not allowed. If each block represents each slice or moment of the present universe then the total loaf will be 'all the time' elapsed in this universe as of now. But, the question is - Is there any way in which there should be a linking parameter from one block to another like a map with a link to each specified events evolution through time. Let's make the past evolution as $A$, present as $P$, future as $B$ thus the mapping that can be achieved as;

$$
\tau=A \stackrel{+T}{\rightarrow} P \stackrel{+T}{\rightarrow} B
$$

Where $\tau$ is the mapping function and $+T$ is the flow of time. Note, that $+T$ is same between $A$ and $P$ as to $P$ and B. But, this $+T$ remains constant unless we apply some relativistic norms as to the delay/fasten of time $b y$ means of the dilation factor of relativity as such;

$$
\Delta T=\frac{\Delta t}{\sqrt{1-\frac{v^{2}}{c^{2}}}}
$$

Where $\Delta T$ is the change in time. But still something is missing from the scenario that we will encounter in the next section.

TEMPORAL MONODROMY - The formal question that arises in our mind is that, can we hold a piece of time? Just like we hold a piece of space? By piece of space, I should say that a chunk of space containing the 3-known dimensions length, breadth and height. The answer is obviously 'no'. No, we can't hold time, it just slips away, this could be the fact that our spatial plane is complex rather than simple and there is a helicoids motion of our space that takes place surrounding the origin of time, in such a way as through, nature protects the time and prevents the humans to get hold of it by sweeping away revolving around the centre points. And that's in physics can be termed as a monodromy. And I have introduced here the concept of temporal monodromy. Let's, say that there is an open space $\mathcal{O}$ which has a subset $\mathcal{O}_{s}$ through a punctured complex plane $\mathbb{C} \backslash\{0\}$ where some analytic function $f(z)$ goes around and continued back into $\mathcal{O}_{s}$. This can be represented as;

$$
\begin{gathered}
f(z)=\log (z) \\
\mathcal{O}_{s}=\{z \in \mathbb{C} \mid \operatorname{Re}(z)>0\},|z|=1
\end{gathered}
$$

Through one complete rotation, the function of the analytic continuation won't come back to its starting point $f(z)$ but will return to $f(z)+2 \pi i$. Thus we can get a complete equation of the helicoids whose radius if can be defined by $\sigma_{R}$ revolving around an angle $\vartheta$ over a height $h$, then the area of revolution is generated as;

$$
\begin{aligned}
& \frac{\vartheta}{2}\left[\sigma_{R} \sqrt{\sigma_{R}^{2}+\left(\frac{h}{\vartheta}\right)^{2}}\right. \\
& \left.+\left(\frac{h}{\vartheta}\right)^{2} \operatorname{In}\left(\frac{\sigma_{R} \sqrt{\sigma_{R}^{2}+\left(\frac{h}{\vartheta}\right)^{2}}}{\frac{h}{\vartheta}}\right)\right]
\end{aligned}
$$

This in turn modified the 3dimensional of the cylindrical bread into a different coordinates as to say;

$$
\begin{gathered}
x=\rho \cos (\beta \vartheta) \\
y=\rho \sin (\beta \vartheta) \\
x=\vartheta
\end{gathered}
$$

Thus we are ending up with a helicoids slice concept instead of a bread slice concept, but whatever, the concepts can hold and monodromy is special in the sense, the nature always protects the core origin of time from being misaligned by humans as to say in the language of Prof. S.W. Hawking "Nature makes history safe for historians".

\section{TEMPORAL EXPONENTIALITY -}

The very first point that we humans have to know, that, if nature is really a byproduct of an augmented reality, then who exactly is making the simulation? Is there a highly powerful entity existing either in our nature or beyond our nature who with all his super-powered super-intelligent computers are making the simulations, or is this our future humans from future Earth creating all these simulations with their very advanced computers and their processing powers? To answer, the second probability, as we have already understood the simultaneity of past, present and future, we can certainly state that, those humans with highly powerful computers as portrayed in the movie $L U C Y^{n}$ must have developed a way as to break the $2^{\text {nd }}$ law of thermodynamics as they have to proceed behind time to make the nature simulated from the very onset of Big Bang. It is highly probable that, they might create this to solve the problem of existential crisis or it can be due to some unknown reasons that the today's society being far lag in technological advances than them is unable to comprehend. If the first proposition is true that there exists an higher entity that can simulate the entire world of ours, then this might be possible provided we all are playing games inside a giant computer screen which we assumes as our world. But, its not an easy task to create such simulations, and a very rather tremendous computing power is needed to simulate the entire world. For, this whoever, is performing the simulation through his machines must have cracked the $P$ vs. NP conjectu$\mathrm{re}^{\circ}$ as because the nested loops along with the inputs turned out to be exponential if all the parameters rather billions, even more than that has to be taken into account to simulate this whole world. Exponentiality is a major factor that needs to be into consideration if something has to be simulated because without exponentiality, the reality can't be portrayed flawless, so that we humans can in no way understand, our surroundings are also simu- 
lations $^{\mathrm{p}, \mathrm{q}}$. To simulate each inputs there arises many nested loops and in each depth of nested loops there are many more nested loops which tends to make the simulation parameter as;

$$
\text { inputs }^{\text {nested loops }} \cong N P
$$

As this is in the domain of NonPolynomial, so, either they have cracked the puzzle of NP or they have inducted software's and algorithms to convert NP into $P$. this is a very advanced state that we are thinking about.

One thing that needs to be mentioned is that, whether, the simulation is free from any glitches. The glitch can occur in the form of hang, lag, where the machines have to debug. But, if that's the case then how should we discover those glitches. This can be done by monitoring the 4 fundamental forces of nature with an alteration of a billionth or rather more to them in a minute way, where the four fundamental forces whose changes needs to be taken care of are ;

[1] The strong nuclear force.

[2] The weak nuclear force.

[3] The electromagnetic force.

[4] The gravitational force.

But it should be noted that, any tiny fraction of a changes, maybe it sounds billionth or even more, there would be disaster in this universe and the whole universe will be shattered. So, this arouses a problem that, 'as far as we can't see any forces of nature changing' - does this mean there can be advanced software's that can run without any lag or errors for eternity. Although this sounds unbelievable, this can be the fact, if we are considering a very highly-sophisticated computers to work on the simulations algorithm. Or, perhaps our hypothesis of glitches is totally wrong, glitches are always occurring but maybe in higher order dimensions in the form of perturbations that we humans couldn't be capable of noticing at all. But, why higher order dimensions are more perturbed to glitches? This arises from the fact that in high dimensions, the degrees of freedom are more, therefore, if the errors really made any impact on the simulated reality then their affects are more observed in the higher order dimensions than the lower order dimensions where its almost nil.

Every physicists should be careful about the errors to observe in the system (that is world) if its simulated as, a small error if appeared in reality then this exponentialize into large errors or errors will amplify errors which would result in a catastrophe. So, this could be a possible indication that simulation if there, has been very precise and error-free as any minute mistake will alter the reality and make it an unstable state of vacua.

MANDELA EFFECT \& DÉJÃ VU Some things are difficult to prove because of the unseen enormous complexity lying beneath them to perceive. Two such things are the Mandela effect ${ }^{\mathrm{s}}$ and Déjà $v u^{t}$ where its impossible to use the fundamental physical law to prove their identities. The only option that remains to humans is to watch the glitches. This thing which appears to the people as a worldwide phenomenon, its also very hard to ignore its effects on physics and so, physicists have wondered this with the simulation hypothesis or even with the existence of a parallel world. The existence of the parallel world has come to the scientific community when a CMBR (Cosmic Microwave Background Radiation) spotted on the south-eastern hemisphere of the COBE satellite data as a cold blue spot. Many theorists have started to believe that this cold spot is the result of a washed away radiation zone due to the passing by of a parallel universe in the multiverse void. String theorists have already been saying the possibility of multiple universe a decades ago. So, this is just a missing piece to the puzzle. But, although this fascinates the theorists especially to some astrophysicists this is just a source of distraction from pure physics.

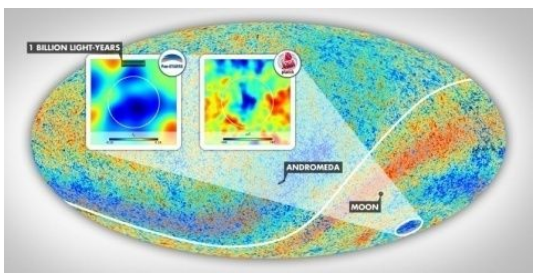

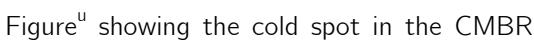
anisotropy data of the early universe ${ }^{v}$.

All over the internet, there are numerous examples of Déjà vu as just a pure psychological thing without any resemblance to any of this and Mandela effect as a source of confabulation or false memory ${ }^{\mathrm{w}}$ happening in large numbers of people. Many even argued that those who dealt with such theories of parallel world, intersecting timelines, and multiple universes are spreading conspiracy theories but what can say, someday these conspiracy theories will solve the puzzle when a glitch in the simulation will become observable to the scientific community as an evidential identity.

If Moore's law ${ }^{x}$ holds true and if there really exists some higher order entities who are simulating us then both are making sense, as there are possibilities of other entities existent in our universe that are controlling us and we are not even near to their technological advancement. Or, if in future, some $10,000 \mathrm{yrs}$ later to be precise - just as an approximate there can be technological development so advanced that those humans would prefer to simulated the whole timeline of the universe including their own (which is quite obvious) and we are just a byproduct of this simulated entries. But why they would choose to simulate the entire universes timeline is a question of mystery - perhaps this can be the fact that they are escaping to a higher order reality and they don't want the timeline of the universe to get hampered by making a gap in the present reality - hence simulated everything from start to end in order to maintain a balance of the universe. Or, maybe they want to see the fate of the universe by simulating the entire timeline.

${ }^{2}$ Project Director of AATWRI-R\&D Electro-Gravitation Simulation \& Propulsion Laboratory, Bhubeneshwar, Odisha, India.

${ }^{3}$ Author declares that he does not have any conflicting interests as related to this paper. 
a'D'Inverno, R., \& Vickers, J. (2021). Introducing Einstein's Relativity: A Deeper Understanding (2nd ed.). Oxford University Press.

${ }^{\mathrm{b}}$ Taylor, W., \& Wang, Y. N. (2015). The F-theory geometry with most flux vacua. Journal of High Energy Physics, 2015(12), 121. https://doi.org/10.1007/jhep12(2015)164

${ }^{\mathrm{c} B h a t t a c h a r j e e, ~ D ., ~ \& ~ B h a t t a c h a r j e e, ~ D . ~(2021) . ~} 17$ Essays On Modern Physics: From Sonoluminescence To Superconductivity. Authorea Preprint. Published. https://doi.org/10.22541/au.162998994.41631106/v1

${ }^{d}$ Hawking, S. W. (1992c). Chronology protection conjecture. Physical Review D, 46(2), 603-611.

https://doi.org/10.1103/physrevd.46.603

eThrone, K. S. (1993). Closed Timelike Curves. A Caltech Goldenrod Preprint in Theoretical Astrophysics or Gravitational Physics. Published.

${ }^{f}$ Harikant, A., Singha Roy, S., \& Bhattacharjee, D. (2021c). Computing the temporal intervals by making a Throne-Morris wormhole from a Kerr black hole in the context of $f(R, T)$ gravity. International Journal of Scientific Research and Management, 9(07), 72-92. https://doi.org/10.18535/ijsrm/v9i07.aa01

${ }^{9}$ Stewart, I. (2010c). Grandfather paradox. Nature, 464(7293), 1398. https://doi.org/10.1038/4641398a

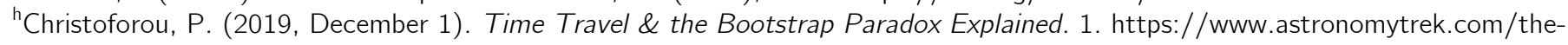
bootstrap-paradox-explained/

'Davis, M. (2020, December 23). 7. Polchinski's paradox. Big Think. https://bigthink.com/surprising-science/10-bizarreparadoxes? rebelltitem $=8$

jS. (2021b). The Cosmic Landscape bySusskind. Back Bay Books.

${ }^{k}$ Intuition behind $\$ \mid z e t a(-1) \$=\$ \backslash$ frac $\{-1\}\{12\} \$$. (2017, January 6). Mathematics Stack Exchange.

https://math.stackexchange.com/questions/2086035/intuition-behind-zeta-1-frac-112/2086052

'Greene, B. (2010). The Elegant Universe: Superstrings, Hidden Dimensions, and the Quest for the Ultimate Theory (2nd ed.). W. W. Norton \& Company.

mJentsch, C., Politis, D. N., \& Paparoditis, E. (2014). Block Bootstrap Theory for Multivariate Integrated and Cointegrated Processes. Journal of Time Series Analysis, 36(3), 416-441. https://doi.org/10.1111/jtsa.12088

"Besson, Luc. (Director). (2014). Lucy (Film). EuropaCorp.

${ }^{\circ}$ Gennady, S. (2020). Artificial Mind: The Traveling Salesperson Problem: The P vs. NP Problem. Ridero.

${ }^{\mathrm{P} B o s t r o m, ~ N . ~(2003) . ~ A r e ~ W e ~ L i v i n g ~ i n ~ a ~ C o m p u t e r ~ S i m u l a t i o n ? ~ T h e ~ P h i l o s o p h i c a l ~ Q u a r t e r l y, ~ 53(211), ~} 243-255$.

https://doi.org/10.1111/1467-9213.00309

${ }^{\mathrm{q} B}$ Bhattacharjee, D. (2020g). The Mandela effect, Déjà vu and possible interactions with the parallel world. OSF Preprints - IndiaRxiv. Published. https://doi.org/10.35543/osf.io/gjtfk

${ }^{r}$ Cox, B., Cohen, A., West, S., \& Limited, H. P. (2017). Forces of Nature. HarperCollins Publishers Limited.

sTop Secret Writers. (2020, September 29). The Mandela Effect: An Academic Explanation.

https://www.topsecretwriters.com/2017/11/mandela-effect-academic-explanation/

${ }^{t}$ Clair, M. (1988). Deja Vu. Callaloo, 35, 213. https://doi.org/10.2307/2930944

"Goss, H. (2015, May 20). What Created the Universe's Cold Spot? Air \& Space Magazine.

https://www.airspacemag.com/space/what-created-universes-cold-spot-180955343/

${ }^{\vee}$ This cosmic Cold Spot challenges our current cosmological model. (2017, April 27). Astronomy. Com.

https://astronomy.com/news/2017/04/cold-spot-challenges-cosmological-model

wharris, A. (1996). False memory? False memory syndrome? The so-called false memory syndrome? Psychoanalytic Dialogues, 6(2), 155-187. https://doi.org/10.1080/10481889609539114

Moore's Law Explained. (2021). Investopedia.

https://www.investopedia.com/terms/m/mooreslaw.asp\#:\%7E:text=Moore's\%20Law\%20refers\%20to\%20Moore's,will\%20pay\% 20less $\% 20$ for $\% 20$ them. 